


\title{
Liquid Crystals Made of Highly Polar Compounds
}

\author{
N.V. Madhusudana \\ Raman Research Institute \\ Bangalore 560 080, India
}

Received 29 October, 1998

\begin{abstract}
Liquid crystals are states of matter with symmetries intermediate between those of isotropic liquids and crystalline solids. The role of permanent dipoles in determining the stability of the nematic phase was discussed soon after the discovery of liquid crystals. Though it is now well known that polar interactions are not essential for the stability of the mesophases, strong longitudinal dipoles give rise to many unusual phenomena like reentrant phases, polymorphism of smectic A and nematic liquid crystals, etc. The article gives a summary of these phenomena and phenomenological as well as molecular theories which have been developed to describe them.
\end{abstract}

\section{Introduction}

Thermotropic liquid crystals made of low molecular weight $(\sim 200-400)$ organic compounds were discovered more than 100 years ago. The simplest liquid crystal is the nematic with a long range orientational order of rod like (Fig. 1) or disc-like molecules [1,2]. The average direction of alignment of the molecules is denoted by a dimensionless unit vector called the director, n. The molecular interactions responsible for the stability of the nematic have been the subject of much research activity for a long time. Indeed the first theory was proposed by Max Born in 1916 [3] in which he proposed that the interactions between the permanent dipoles stabilise the orientational order in the medium which would hence have a permanent polarization. The nematic-isotropic (NI) transition would be analogous to a ferroelectric-paraelectric transition and would be second order in nature. Efforts made to detect the polarization did not yield any positive results and further, many compounds like p-quinquephenyl which do not have molecules with net dipole moments were found to exhibit the nematic phase. The N-I transition has also been found to have a first order characteristic in all known cases. Further, all the physical properties of the nematic indicate that the director $\hat{\mathbf{n}}$ is apolar in nature even when the molecules have strong dipole moments. For example, the topological defects of the nematic are half-strength disclinations around which a thin nematic sample exhibits two dark brushes between a pair of crossed polarizers. This implies a continuous rotation of the director by $\pi$ rads around the core of the

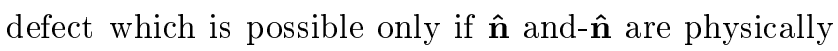
equivalent. As such the orientational order parameter in the nematic is a second rank tensor which for cylindrically symmetric molecules takes the simple form:

$$
S=\frac{3<\cos ^{2} \theta>-1}{2}=<P_{2}(\cos \theta)>
$$

in which $\theta$ is the angle made by the molecular long axis with the director and the angular brackets signify a statistical average. This means that $\mathrm{S}>\mathrm{O}$ and $\mathrm{S}<\mathrm{O}$ will imply very different distribution functions peaking around $\theta=\mathrm{O}$ and $\pi / 2$ rads respectively. As such, in the Landau-deGennes theory of the nematic, the 3rd order term has to be taken into account. The free energy density reads as:

$$
F=\frac{a}{2}\left(T-T^{*}\right) S^{2}-\frac{B}{3} S^{3}+\frac{C}{4} S^{4}+\ldots
$$

in which $\mathrm{T}^{*}$ is a 'hypothetical' second order transition temperature and a,B and $\mathrm{C}$ are the usual temperature independent coefficients. The above form leads to a first order NI transition [1] with

$$
T_{N I}=T^{*}+\frac{2 B^{2}}{9 a C}
$$




\section{Some special properties ex- hibited by compounds with highly polar end groups} $S_{N I}=2 B / 3 C$.

Maier and Saupe [4] developed a molecular theory of nematics in which they proposed that anisotropic dispersion interactions stabilise the liquid crystal. The theory also predicts a first order NI transition.

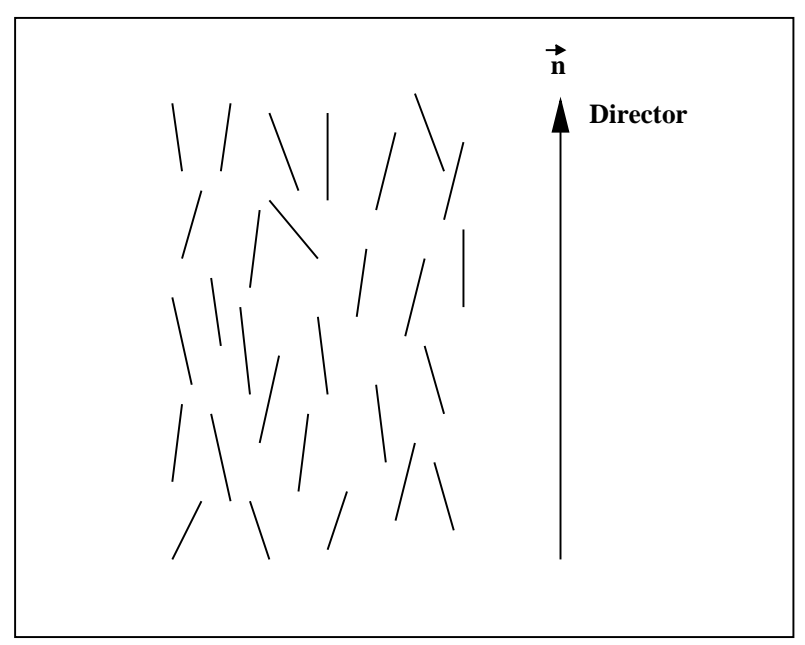

Figure 1. Schematic diagram showing the orientational order of rod like molecules in nematic liquid crystals.

The twisted nematic (TN) liquid crystal display (LCD) was invented by Schadt and Helfrich in 1971 [5] which is the basis of the most successful flat panel display technology worth about 10 billion US $\$$ annually at present. The threshold voltage for the operation of the display is $\alpha(\Delta \epsilon)^{-1 / 2}$ and considerable effort has been expended over the years to increase the dielectric anisotropy $\Delta \epsilon$ in order to reduce the threshold voltage. A large number of compounds with the highly polar cyano-or nitro-end groups have been synthesised. Many of these compounds exhibit unusual phenomena like reentrant phases, smectic A polymorphism etc, which are not seen in weakly polar compounds. Obviously, dipolar interactions play an important role if the dipole moments are large enough. Both phenomenological and molecular theories have since been developed to describe these phenomena. We shall summarise these developments in the following sections. (a) Dielectric properties and antiferroelectric short range order

One of the first measurements made on compounds with cyano end groups was naturally on their dielectric constants. Schadt [6] found that there was an unusual feature in the measured values: The average dielectric constant in the nematic phase is $\bar{\epsilon}=\left(\epsilon_{11}+2 \epsilon_{\perp}\right) / 3$. As the density has a small jump $(\sim 0.5 \%)$ to a lower value across the NI transition, one would expect that $\epsilon_{i s}$ would also be smaller than $\bar{\epsilon}$ in the nematic phase by a similar magnitude. Instead, Schadt found that $\bar{\epsilon}$ had a positive jump by $\sim 2 \%$ across $T_{N I}$ (Fig. 2). We [8] interpreted this to mean that the medium has an antiferroelectric short range order. The dipolar interaction energy is $\sim \mu^{2} / r^{3}$ where is $r$ is an appropriate intermolecular distance and is strongest when the long molecules are side by side with $r \sim$ the molecular diameter $(\simeq 5 \AA)$. In this 'side on' position, the antiferroelectric alignment is energetically favoured. Moreover in this configuration, the number of neighbours of any given molecule is $\sim 6$. On the other hand, in the end to end configuration favouring ferroelectric alignment the number of neighbours is $\sim 2$, and further the interaction energy is much weaker as $r$ is now $\sim$ the length of the molecule $(\sim 25 \AA)$. The antiparallel near neighbour configuration leads to the apolar director $\hat{\mathbf{n}}$, unlike the assumption made by Born. However, we do not expect an antiparallel long range order in the liquid nematic medium. The problem was discussed $[8,9]$ using the Bethe cluster approximation. The orientational part of the intermolecular potential energy is assumed to be of the form

$$
V_{i j}\left(\theta_{i j}\right)=A P_{1}\left(\cos \theta_{i j}\right)-B P_{2}\left(\cos \theta_{i j}\right)
$$

where $P_{1}$ and $P_{2}$ are the first and second Legendre polynomials respectively. The positive sign of the $P_{1}$ term favours an antiparallel orientation and the negative sign of the $P_{2}$ term arises from the anisotropic dispersion interaction. Each molecule is supposed to be surrounded by $z$ nearest neighbours, no two nearest neighbours being considered as nearest neighbours of 
each other. Each 'outer' molecule $j$ of the $(z+1)-$ molecule cluster is also supposed to be subjected to a mean field potential

$$
V_{j}=-B^{*} P_{2}\left(\cos \theta_{j}\right) .
$$

Self consistency requires that $\left\langle P_{2}\left(\cos \theta_{j}\right)\right\rangle$ should be independent of whether the molecule is considered to be a central or an outer molecule in the cluster. It is calculated using the appropriate Boltzmann probability distribution for the configurations of the cluster. The free energy of the medium is also calculated using the relevant partition functions to evaluate the NI transition point. The short range order parameters $<P_{1}\left(\cos \theta_{i j}\right)>$ and $<P_{2}\left(\cos \theta_{i j}\right)>$ do not vanish even in the isotropic phase but their magnitudes jump to lower values above $T_{N I}$ (Fig. 3 ). The dielectric constants have also been calculated using this model and the negative jump in the antiparallel short range order leads to a positive jump in $\bar{\epsilon}$ as this type of short range order lowers the orientation contribution of permanent dipoles to the dielectric constant.

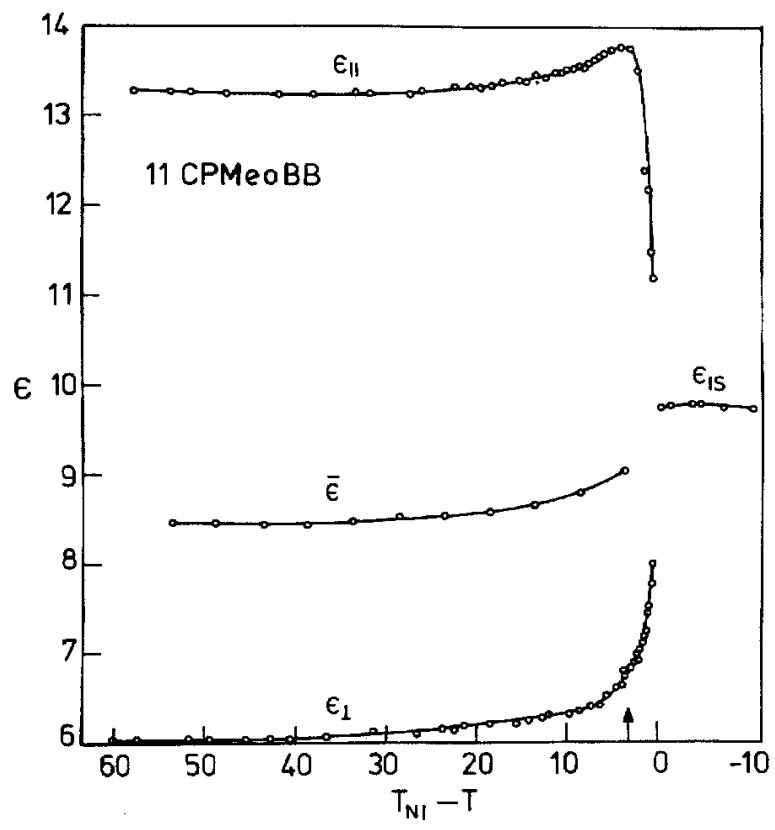

Figure 2. Dielectric constants $\epsilon_{11}$ and $\epsilon_{\perp}$ and the mean value $\bar{\epsilon}$ in the liquid crystalline phases and $\epsilon_{i s}$ in the isotropic phase of the highly polar compound 4-cyanophenyl-3'-methoxy-4 (4'-n-undecylbenzoyloxy) benzoate (11 CPMeOBB) which clearly shows a large jump between $\bar{\epsilon}$ and $\epsilon_{i s}$ at the NI transition point. The vertical arrow on the temperature axis indicates the $\mathrm{N}-\mathrm{A}_{d}$ transition temperature [7].

The antiparallel short range order was later confirmed by Xray scattering measurements on highly po- lar compounds like pentyl cyanobiphenyl (5CB) and octyl cyanobiphenyl (8CB). While 5CB exhibits only a nematic phase between the crystalline and isotropic phases, $8 \mathrm{CB}$ exhibits an additional smectic A phase (A) as well. In the A phase, the rod like molecules are arranged in layers such that there is a one dimensional periodicity along the director $\hat{\mathbf{n}}$. The layer spacing in most compounds is close to the molecular length $l$. However, in $8 \mathrm{CB}$ it was found that the layer spacing $d \simeq 1.4 l$, i.e, considerably longer than and incommensurate with the molecular length [10]. This can be understood if neighbouring $8 \mathrm{CB}$ molecules have an antiparallel orientation such that the aromatic moieties of the two molecules overlap (Fig. 4). This structure arises because the strongest attractive interaction between the two molecules is the anisotropic dispersion potential between the highly polarizable aromatic parts which have conjugated electrons. The antiparallel orientation would then throw the alkyl chains which are present at one end of each molecule in opposite directions as shown in Fig. 4. It is indeed likely that two polar molecules form antiparallel 'pairs'. A third molecule would have a frustrated polar interaction with such a pair, which might actually favour the formation of such pairs. The structure of these smectic layers is also referred to as 'partial bilayer' as the spacing $d$ satisfies $l<d<2 l$ and the $\mathrm{A}$ phase is called the $\mathrm{A}_{d}$ phase. Indeed such compounds are found to exhibit strong smectic A like short range order even if there is no long range smectic order, as in the case of $5 \mathrm{CB}$.

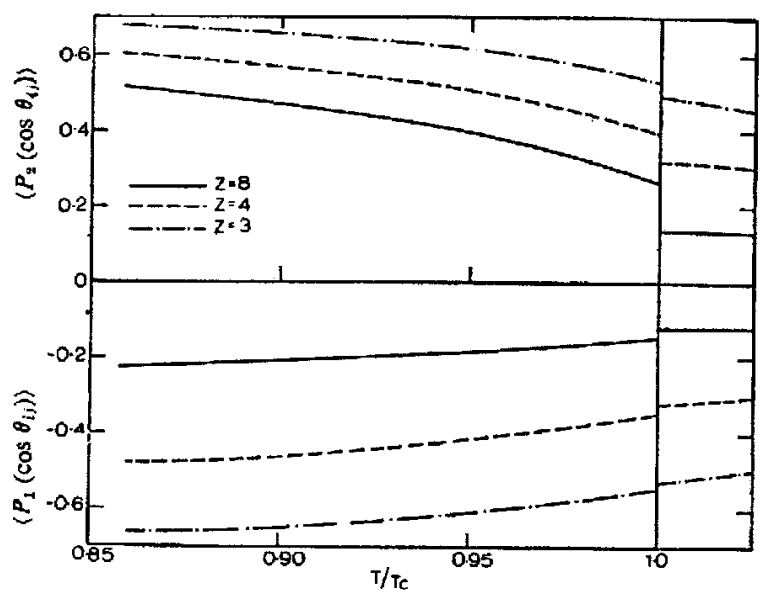

Figure 3. Short range order parameters $\left\langle P_{2}\left(\cos \theta_{i j}\right)\right\rangle$ and $\left\langle P_{1}\left(\cos \theta_{i j}\right)>\right.$ as functions of the reduced temperature $\mathrm{T} / \mathrm{T}_{c}$ for different values of the number of nearest neighbours $z(\mathrm{~A} / \mathrm{B}=0.5)[9]$. 


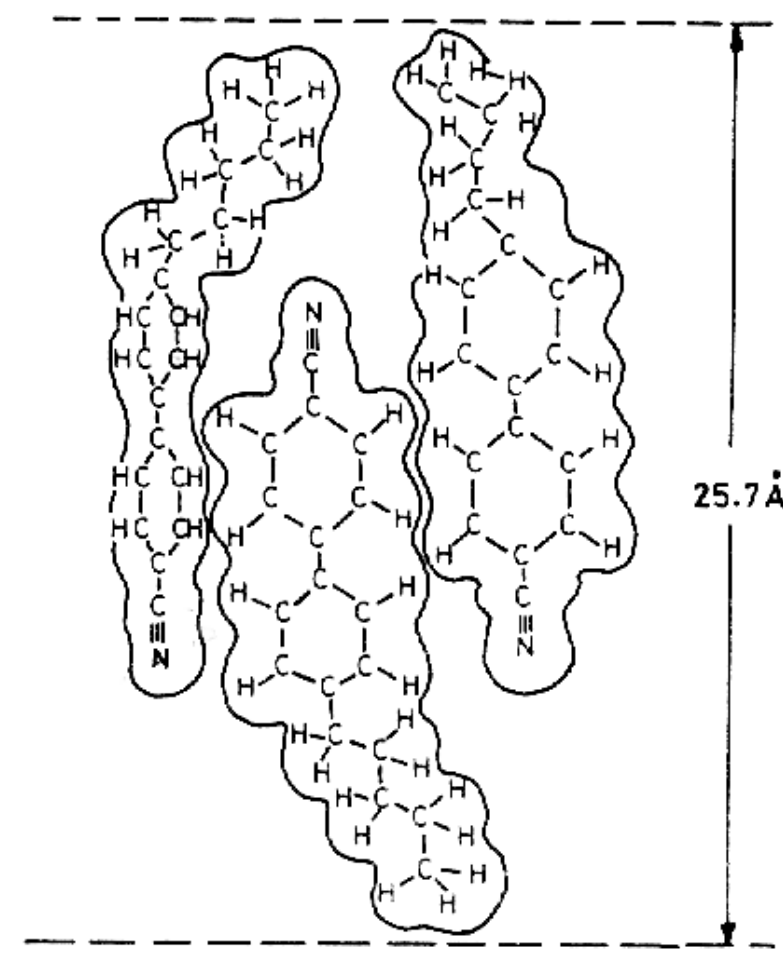

Figure 4. Schematic diagram of the arrangement of neighbouring 5CB molecules showing the origin of a length which is considerably longer than the molecular length [10].

\section{(b) Reentrant liquid crystalline phases:}

A new type of phase sequence among liquid crystalline phases was discovered by Cladis in 1975 [11] when she found that in some composition ranges, a binary mixture of compounds with cyano-end groups exhibited the following phase sequence on cooling: isotropic-nematic - smectic A - reentrant nematic. As the smectic A phase breaks the translational symmetry of the nematic along the director $\hat{\mathbf{n}}$, it usually occurs only at temperatures below the range of stability of the $\mathrm{N}$ phase. The reemergence of the higher symmetry $\mathrm{N}$ phase at lower temperatures is quite unusual,though reentrant phases are of course known in ferroelectric systems and superconductor-normal metal phase transitions etc. [12] Subsequent to the discovery, many single compounds whose molecules have the polar cyano end group have been found to exhibit reentrant phases $[13,14]$. In particular, many compounds are known to exhibit a double reentrant sequence in which below the temperature range of stability of the reentrant nematic phase, a second reentrant smectic A phase is found to occur as well [14]. X ray investigations [15] have shown that the reentrant smectic A phase has a monomolecular layer spacing i.e, $d \simeq l$. Indeed one compound is known to exhibit a triple reentrance in which two reen- trant nematic as well as two reentrant smectic A phases have been found [16].

\section{(c) Smectic A polymorphism:}

Other remarkable phenomena have been found in compounds with the strongly polar cyano - or nitro-end groups. For example, 4-n-pentylphenyl-4' $4^{\prime \prime}$ - cyano benzoyloxy) benzoate (DB5 for short) has two ester linkage groups in the aromatic core such that their dipole moments are essentially antiparallel to that of the cyano end group. Sigaud et al [17] found that some binary mixtures of this compound with the weakly polar compound terephthal bis butylaniline (TBBA) exhibit a first order transition from a monolayer $\left(\mathrm{A}_{1}\right)$ to a bilayer $A_{2}$ phase as the temperature is lowered. In a structurally related compound called 4-decylphenyl $-3^{\prime}$-methyl-4' (4" - cyano benzoloxy) benzoate (10PM$\mathrm{CBB}$ ), a first order transition is found from an $\mathrm{A}_{d}$ phase occurring at higher temperatures (with $d \simeq 1.6 l$ ) to an $\mathrm{A}_{2}$ phase in which two Bragg diffraction spots corresponding to both $l$ and $2 l$ are condensed [18] (Fig. 5).

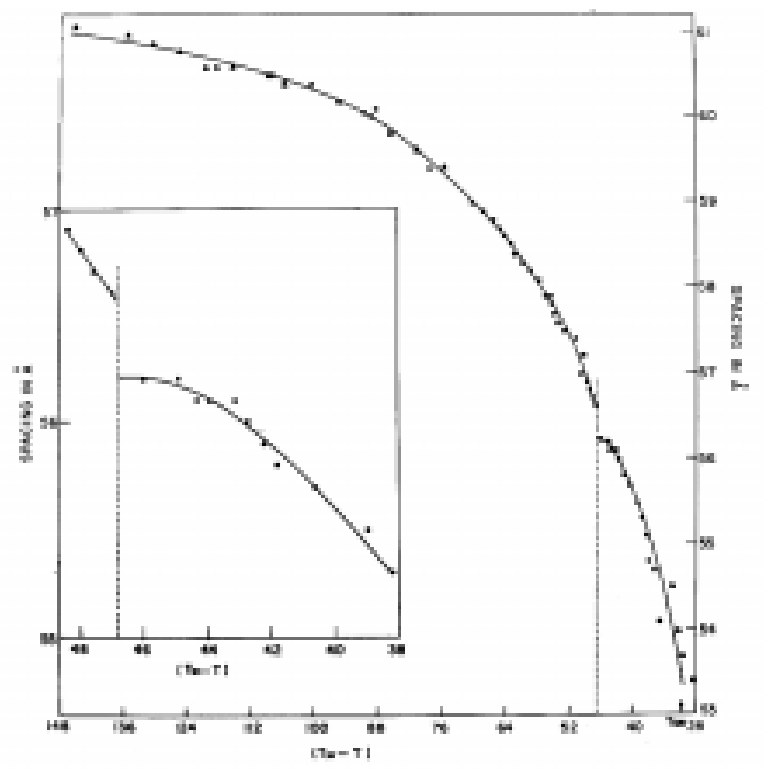

Figure 5. Temperature variation of the layer spacing of 10PMCBB. The $\mathrm{A}_{d}-\mathrm{A}_{2}$ transition occurs with a jump in the layer spacing at the temperature corresponding to the dashed line. The region around this transition point is shown on a magnified scale in the inset[18].

Yet another type of smectic A known as the antiphase $\tilde{A}$ is found in some pure compounds and mixtures. For example, some mixtures of DB5 and 4'- (4n-pentyl stylyl) phenyl- $4^{\prime \prime}$-cyanobenzoate (C5 stibene) 
exhibit a high temperature $A_{1}$ phase with a diffuse scattering at a wavevector which is split such that its $\mathrm{z}$ component is half of $(2 \pi / d)$. On cooling the mixture, a phase transition results in which the diffuse spots get condensed. A superlattice long range order develops in a lateral direction, with a spacing of $\sim 130 \AA$. On further cooling, this goes over to an $\mathrm{A}_{2}$ phase. Thus the intermediate phase occurring between $A_{1}$ and $A_{2}$ phases is an antiphase (Fig. 6) in which the local $\mathrm{A}_{2}$ like regions change along the layers such that the polar head group rich and aliphatic chain rich regions alternate spatially in a regular manner [19].

From the point of view of molecular structures, the compounds which exhibit reentrant nematic and smectic phase have additional dipoles in the aromatic moieties which are parallel to the dipole of the cyano or nitro end group (as in n-CPMBB, Fig. 7 ). On the other hand in compounds exhibiting $\mathrm{A}_{2}$ and $\tilde{A}$ phases the additional dipoles are oriented in the opposite direction (as in nPMCBB, Fig.7). Thus in all these cases, the dipolar interactions do play a significant role in determining the nature of the liquid crystalline phases exhibited.

Several theoretical models have been developed to account for the rich variety of liquid crystalline phases exhibited by the highly polar compounds. We briefly outline them is the next section.

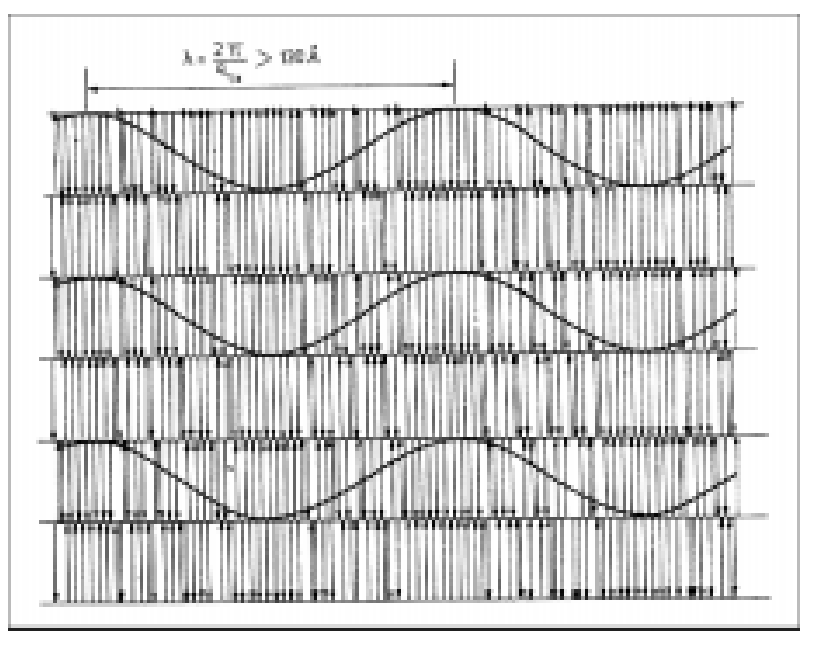

Figure 6. Schematic diagram of the arrangement of molecules in the smectic antiphase $\tilde{A}$.

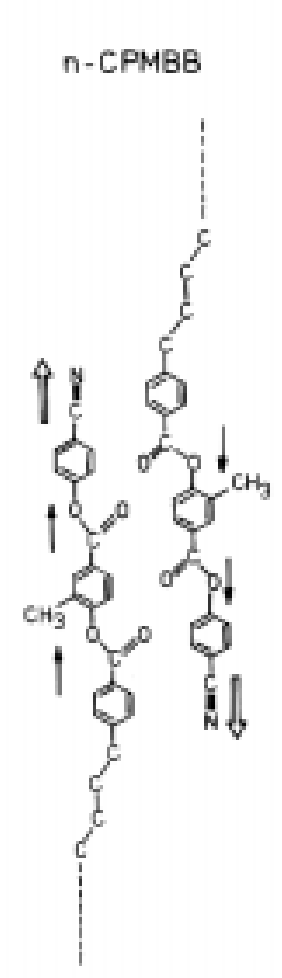

(a) (b)

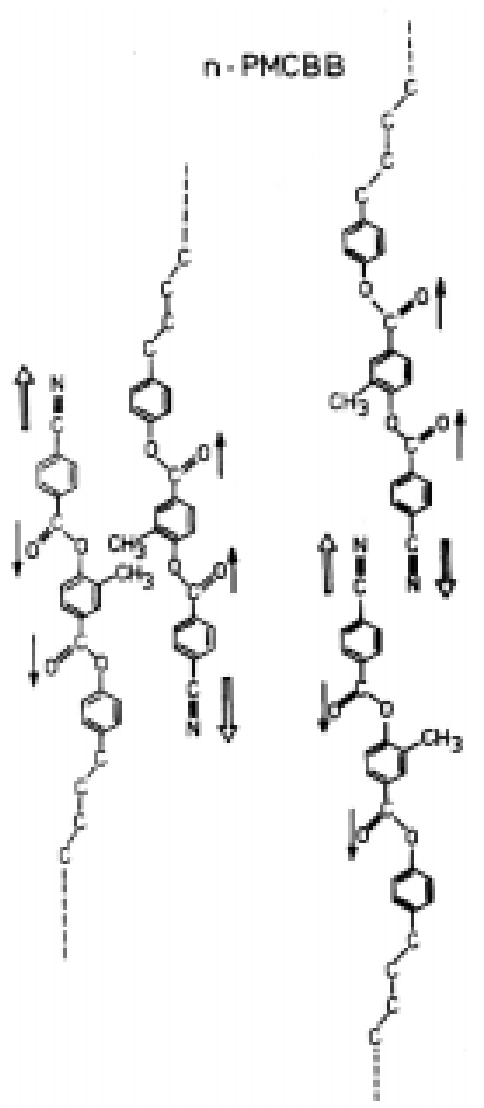

(c)
Figure 7. Schematic diagrams showing the disposition of various dipolar groups of a pair of (a) n-CPMBB molecules favouring the occurrence of reentrant phases. In (b), an overlap of the aromatic cores of two n-PMCBB molecules is not favoured by the dipolar interactions and hence leads to the structure shown in (c), which gives rise to $\tilde{A}$ and $\mathrm{A}_{2}$ phases [18].

\section{Theoretical models}

Both phenomenological Landau theories and molecular theories in which specific intermolecular interactions have been taken into account have been proposed to account for the phenomena described above. We shall first briefly describe the highly successful Landau theory developed by Prost and coworkers.

\section{(a) Prost's phenomenological theory:}

As we have described earlier, the antiferroelectric short range order in the highly polar compounds effectively generates a length which is longer than a molecular length (See Fig. 4). In the smectic $\mathrm{A}_{d}$ phase the layers condense to develop a 1-D translational order with 
a partial bilayer spacing. The exotic phenomena exhibited by many highly polar compounds clearly show that there is a competition between this length and other lengths, leading to frustration. Prost argued that the 'natural' length is the molecular length $l[1,20]$. The Landau free energy density is then expanded in terms of the two order parameters $\rho$ and $\phi$ corresponding to these two lengths,

$$
\phi=\frac{1}{\sqrt{2}}\left(\psi_{1} e^{i \hat{q} \cdot \hat{r}}+C C\right)
$$

and

$$
\rho=\frac{1}{\sqrt{2}}\left(\psi_{2} e^{i \hat{q} \cdot \hat{r}}+C C\right)
$$

in which $\phi$ corresponds to the partial bilayer order and $\rho$ to the monomolecular order, $\psi_{1}$ and $\psi_{2}$ are the corresponding amplitudes and $\vec{q}$ is the wavevector of the $1-\mathrm{D}$ density wave in the smectic A phase. An appropriate form of the free energy density is given by

$$
\begin{aligned}
f= & \quad \frac{A_{1}}{2} \phi^{2}+\frac{C_{1}}{4} \phi^{4}+\frac{A_{2}}{2} \rho^{2}+\frac{C_{2}}{4} \rho^{4} \\
+ & A_{12} \phi \rho-\frac{B_{12}}{\sqrt{2}} \phi^{2} \rho+\frac{C_{12}}{3} \rho^{2} \phi^{2} .
\end{aligned}
$$

As the two order parameters correspond to two different periodicities, it is appropriate to write

$$
\begin{aligned}
& A_{1} \phi^{2}=a_{1} \phi^{2}+A_{1}^{\prime \prime}\left(\left(\Delta+q_{1}^{2}\right) \phi\right)^{2} \\
& \text { and } \\
& A_{2} \rho^{2}=a_{2} \rho^{2}+A_{2}^{\prime \prime}\left(\left(\Delta+q_{2}^{2}\right) \rho\right)^{2}
\end{aligned}
$$

where

$$
a_{1}=\left(T-T_{1}^{*}\right), \quad a_{2}=\left(T-T_{2}^{*}\right)
$$

$\Delta=\partial^{2} / \partial z^{2}$ if the layers are condensed along the $Z$ axis and $q_{1}=2 \pi / l_{d}, q_{2}=2 \pi / l_{1}$, ensuring that $\phi$ condenses with a wavevector $q_{1}$, and $\rho$ with $q_{2}$. It is clear that this very general expansion can give rise to many different structures depending on the relative importance of the coupling terms [1].

In the smectic $A_{1}$ phase, $l_{d} \simeq l_{1}$ and the $(\rho \phi)$ term is the dominant one. In the smectic $A_{2}$ phase, $l_{d} \simeq$ $2 l$ which results from the domination of the $\phi^{2} \rho$ term, while the $(\rho \phi)$ term is negligible. If there is some incommensurability between the wavevectors corresponding to $l_{d}$ and $2 l$, phase matching can give rise to the antiphase $\tilde{A}$ with $\rho\left(2 q_{z}\right)$ and $\phi\left(-q_{z}, q_{x}\right), \phi\left(-q_{z},-q_{x}\right)$ oriented as shown in Fig. 8. Another interesting prediction of this model is the possible existence of an incommensurate smectic phase in which two collinear incommensurate wavelengths can coexist. The double reentrant behaviour is also predicted in an appropriate parameters space [1]. Many other features, like $A_{1}-A_{d}$ critical point etc, predicted by this model have been experimentally realised (See Ref [1] for a comprehensive review).

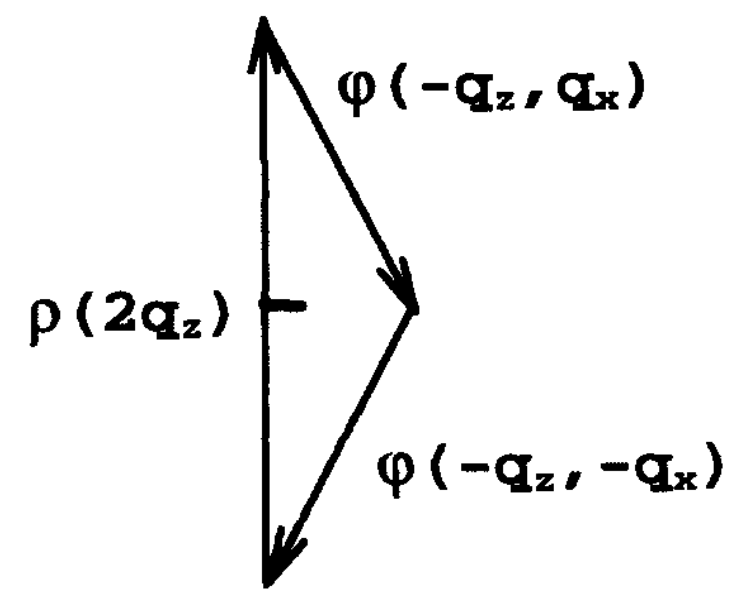

Figure 8. Schematic diagram showing the development of lateral components of the wavevector corresponding to the antiparallel order $\phi$ to ensure a phase matching with the wavevector of the mass density which has only a zcomponent[1].

The basic assumption of the phenomenological model is the possibility of condensation of the smectic layers at the two length $l_{1}$ and $l_{d}$. While the length $l_{d}$ is easy to justify, the monomolecular length, which is simply assumed to be the 'natural' length of the molecule is not so easy to justify, as we shall discuss in the next section.

(b) Molecular theories of the liquid crystalline phases exhibited by highly polar compounds:

Several ideas have been put forward to account for the molecular origin of the exotic phase transitions exhibited by compounds made of highly polar molecules. Cladis herself put forward a physical model in which an efficient packing of the antiparallel pairs is the main driving mechanism [12]. Such pairs look like 'rolling pins' (Fig. 9) which are fat in the middle. As the temperature is lowered in the $\mathrm{A}_{d}$ phase, the concentration 
of dimers as well as the density of the medium increase and the packing of the dimers is no longer efficient. The entropy of the medium can be increased if the dimers slip out of the layers leading to the reentrant nematic phase. On further increase in the density, the molecular chains in successive layers can overlap leading effectively to the 'monomolecular' smectic $\mathrm{A}_{1}$ phase. A detailed molecular theory based on this idea was later developed by Saha et al [21]. However, it is clear that the $\mathrm{A}_{d}$ phase in this picture is so tightly packed that the layers are unlikely to be fluid.

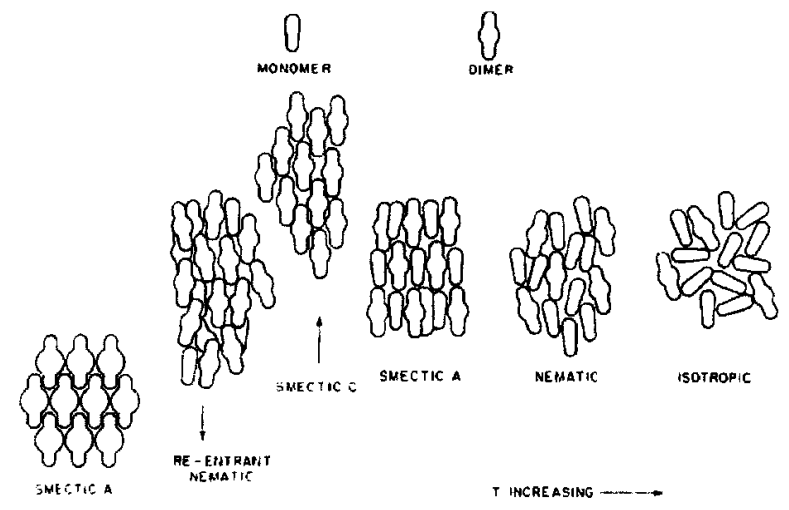

Figure 9. Schematic diagram showing the molecular packing of dimers and monomers in different phases as proposed by Cladis [12].

Longa and de Jeu [22] developed a model in which the dimers break up at elevated temperatures so that there is a mixture of monomers and dimers. This would lead to a preponderance of monomers in the higher temperature phases, while experimentally the $\mathrm{A}_{1}$ phase occurs at low temperatures. As such this mechanism is unlikely to be responsible for the observed phenomena.

Berker and co-workers [23] have developed a model called the 'frustrated spin gas' model in analogy with a similar model used in describing magnetic systems. The basic idea is that if there is an antiparallel pair, the orientation of a third molecule in its vicinity is 'frustrated' as it would be parallel with one molecule of the pair while antiparallel with the other. Various possibilities of relieving this frustration give rise to the observed phases. The configurations of triplets of molecules are needed to see the effect of the frustration. The molecules which are assumed to be aligned perpendicular to a layer (with perfect orientational order) are allowed to have translational degrees of freedom perpendicular to the layer but with notches in the intermolecular potential arising from the details of the molecular structure, including the zig-zag conformation of the chain. The free energy of the system can be calculated by mapping the problem to the frustrated spin gas model and if the given triplet configuration can have a long range correlation in the layer, it is assumed to represent the smectic phase. If the correlation of the triplets is not extended, it is assumed that the molecules can slip out of the layer i.e, it represents the nematic phase. There are enough degrees of freedom in the model to generate many triplet configurations which are identified with different smectic phases. The model however predicts that the transition to the reentrant $A_{1}$ phase occurs at far too low a temperature.

We now discuss in some detail a simple molecular model proposed by us [24] in which the interactions involving all the specific molecular features of the compounds are taken into account.

We recall that the compounds which exhibit the double reentrant sequence $\mathrm{N} \quad \mathrm{A}_{d} \quad \mathrm{~N}_{R} \mathrm{~A}_{1}$ consist of molecules which have (i) the strongly polar cyano end group which is attached to (ii)the highly polarizable aromatic core with 2 to 4 phenyl rings and (iii) a long enough alkyl chain with 6 or more carbon atoms. As we have already discussed the electrostatic dipolar orientation leads to the antiparallel association between pairs of molecules. The strongest interaction is, however, the dispersion energy between the aromatic cores having high polarizabilities. Further, the effective dipole momment of each molecule is enhanced as the induced dipole due to the field generated by the cyano group of a neighbour is parallel to the permanent dipole of the cyano group of the given molecule. On the other hand, the chains are so far apart that the dispersion interaction between them is negligibly small (Fig.10).

Let us now consider a 'parallel' configuration of the pair as shown in Fig. 10. The dispersion interaction between the aromatic cores is the same as in the antiparallel configuration, but that due to the chains now adds to the attractive energy. The dipolar potential in repulsive, but the effective dipole moment itself is reduced considerably as the induced dipole due to the neighbour is now antiparallel to the permanent dipole of the given molecule. As both the dipole-induced dipole and chain-chain dispersion interactions favouring the parallel configuration vary as $1 / r^{6}$ where $r$ is an 


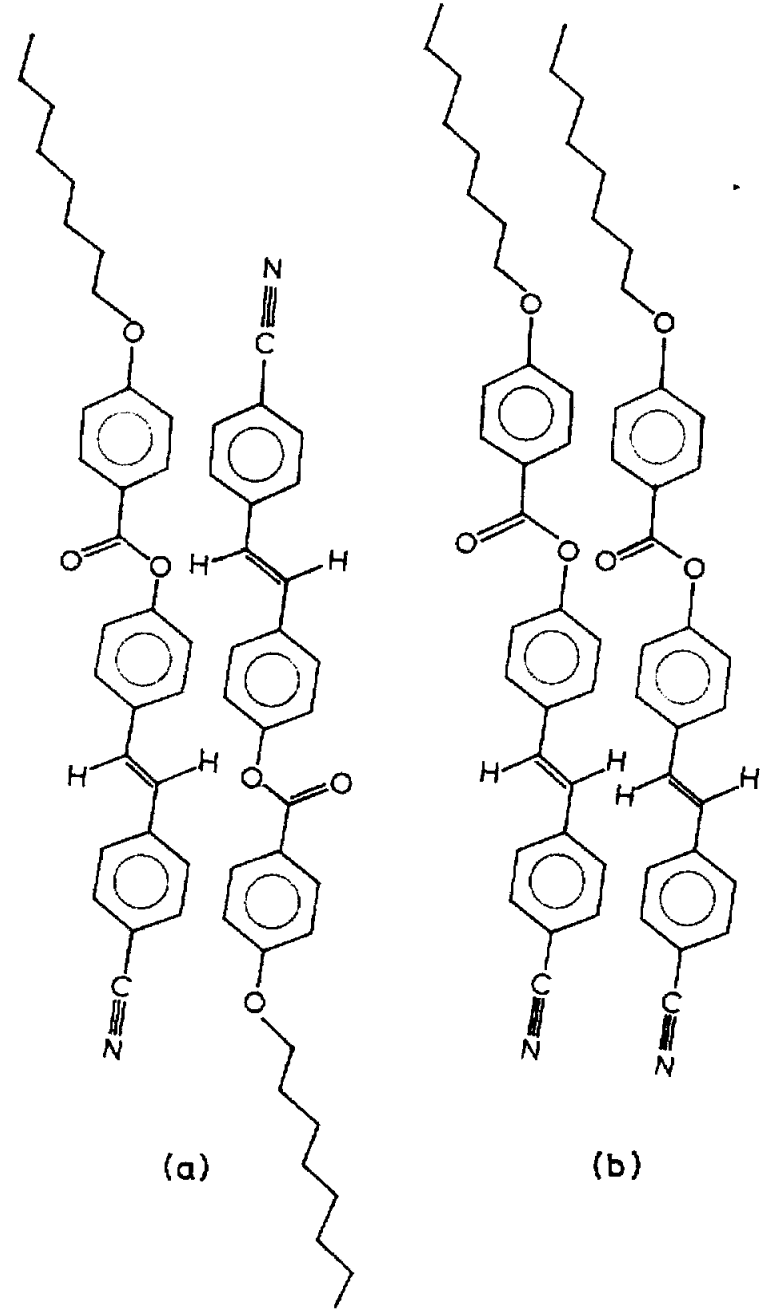

Figure 10. (a) The antiparallel configuration of two octyloxy benzoyloxy caynostilbene (T8) moleculars favoured at moderate intermolecular separations and (b) the parallel configuration favoured at relatively low values of the intermolecular separations.

intermolecular separation, while the permanent dipolepermanent dipole interaction is $\propto 1 / r^{3}$, the parallel configuration can be favoured at sufficiently small values of $r$. The 'configurational' parts of the interaction energy are:

$$
\begin{gathered}
E_{A}=E_{\uparrow \downarrow}=\mu_{n e t}^{2} / r^{3} \\
E_{P}=E_{\uparrow \uparrow}=\mu_{n e t}^{2} / r^{3}-C / r^{6}
\end{gathered}
$$

where $\mu_{\text {net }}=\mu_{\text {perm }}+\mu_{\text {induced }}$ and $C$ the coefficient of the dispersion interaction between the chains. $\mu_{\text {net }}$ $=\mu_{\text {perm }} /\left(1 \mp \chi / r^{3}\right)$ where $\chi$ is the longitudinal component of the polarizability of the aromatic core, the negative (positive) signs corresponding to the antiparallel (parallel) configurations respectively. Calculations with very reasonable values of the parameters show that the parallel configuration is favoured for $r \leq 5 \AA$ in appropriate cases [24]. As the density increases at lower temperatures, the difference in the configurational energy, $\Delta E=E_{A}-E_{P}$ where the subscripts stand for 'antiparallel' and 'parallel' respectively, can be taken to be of the form

$$
\Delta E=R_{1} k_{B} T_{N I}\left(R_{2} / T_{r}-1\right)
$$

in which $\mathrm{R}_{1} k_{B} T_{N I}$ is an interaction parameter expressed in terms of the Boltzmann constant $k_{B}$ and the NI transition temperature $T_{N I} . T_{r}=T / T_{N I}$ is the reduced temperature and $R_{2}$ is the value of $T_{r}$ at which the average intermolecular separation $r$ takes a value such that $\Delta E=0$. The medium is considered to be a mixture of 'A' type and ' $\mathrm{P}$ ' type pairs. The McMillan theory of smectic A phase[2] is then used to calculate the detailed phase diagram in the mean field approximation. As the A type of pairs have chains on both sides the relevant McMillan parameter, which is a measure of the strength of the smectic interaction potential, is given by

$$
\alpha_{A}=2 \exp \left\{-\left[\pi r_{o} /\left(r_{o}+2 c\right)\right]^{2}\right\}
$$

where $r_{o}$ is the length of the aromatic core, $c$ that of the chain and $r_{o}+2 c=l_{d}$ the length of the antiparallel pair. This is considerably larger than the McMillan parameter for the parallel pair which has chains only at one end:

$$
\alpha_{P}=2 \exp \left\{-\left[\pi r_{o} /\left(r_{o}+c\right)\right]^{2}\right\} .
$$

The mean field theory is used to predict a phase diagram as shown in Fig. 11. The $\alpha$ value increases with chain length in a homologous series, and for small values of $\alpha_{A}<.0 .902$, there is a direct transition from the nematic to the smectic phase with $P$ type of pairs, i.e, the $A_{1}$ phase. For $0.902<\propto_{A}<1.088$, we get a double reentrant sequence. As the temperature is raised, $A_{1}$ goes over to a nematic phase, but at higher temperatures, the A type of pairs build up and as the corresponding McMillan parameter is high, the smectic phase which is rich in the A-type of pairs reappears i.e, we get the $A_{d}$ phase. At a high enough temperature, this goes over to the nematic phase. For $\alpha_{A}>1.088$, the $\mathrm{A}_{1}$ phase gradually goes over to the $\mathrm{A}_{d}$ phase. The double reentrance is thus seen only in a narrow range of $\alpha_{A}$ values i.e., only in a few homologues. This fact as well as the detailed parabolic shapes predicted for 
the $\mathrm{A}_{d}-N$ as well as $N_{r}-\mathrm{A}$ phase boundaries are in accord with experimental results [24].

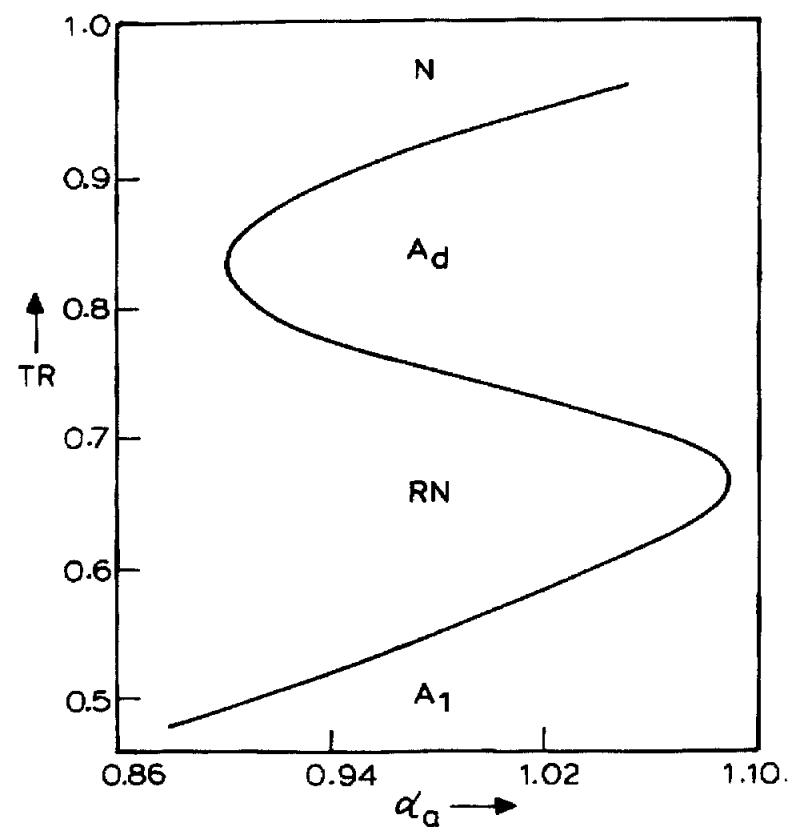

Figure 11. Theoretical phase diagram as a function of $\alpha_{A}$, the McMillan parameter for the antiparallel pairs $\left(R_{1}=10\right.$, $\left.\mathrm{R}_{2}=0.8\right)$.

The model thus gives a natural explanation of the fact that the $A_{1}$ with monolayer spacing accurs at relatively low temperatures while the $A_{d}$ phase with partial bilayer spacing occurs at higher temperatures. Further, in this model the neighbouring molecules are parallel in the low temperature monolayer phase and not arbitrarily oriented. Even though the effective dipole moment of this pair is highly reduced due to the reasons discussed earlier, the parallel orientation would imply a polar short range order. In order to test if this is indeed the case, we undertook investigations on the effect of very high electric fields, up to 600esu on such compounds[25]. The main problem in applying such large fields is that the dissipation due to flow of ions which are always present in the sample leads to a heating of the sample. Durand and co-workers [26] recently solved this problem by applying high field pulses of short duration $(\sim \mu \mathrm{sec})$ and a long interval $(\sim 10 \mathrm{sec})$ between the pulses to prevent heating. We designed a different technique in which the local temperature of the sample is measured using an evaporated nickel thermometer so that the heating effect does not cause any problem [25]. Both the dielectric constant parallel to the director and the conductivity $\sigma_{11}$ are measured using an electrical impedance analysis of the cell. The former is sensitive to the long range orientational order of the medium while the latter is sensitive to the translational order. The phase transition temperatures can be located clearly using these measurements.

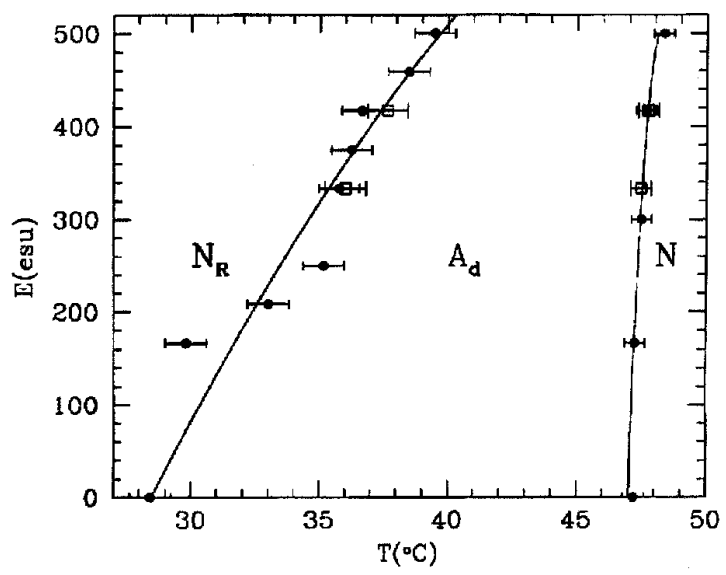

Figure 12. The electric field phase diagram of the binary mixture of hexyloxy cyanobiphenyl and octyloxy cyanobiphenyl with $27 \mathrm{wt} \%$ of the former. Circles are obtained from light scattering measurements and open squres those obtained from the electrical impedance data. The solid lines are guides to the eye. [27]

Using this technique, we have measured the first phase diagram under the action of a strong electric field of a mixture which exhibits the reentrant phase [27] (Fig. 12). Both the $\mathrm{N}-\mathrm{A}_{d}$ and $\mathrm{A}_{d}-\mathrm{N}_{R}$ transition temperatures increase with field, the latter varying more strongly than the former, indicating that the $\mathrm{A}_{d}$ phase will get eventually bounded for a sufficiently large applied field. We can understand this result on the basis of the molecular model mentioned earlier by noting that the electric field produces an increase in the density of the medium. The direct electrostrictive effect gives $\delta \rho$ $=\mathrm{k}_{T} \epsilon_{11} E^{2} /(8 \pi)$ where $\mathrm{k}_{T}$ is the isothermal compressibility. $\delta \rho$ is too small to have any significant effect. However there is a large indirect effect arising from the field induced enhancement of the orientational order, viz,

$$
\delta \rho=\left[\frac{\partial \rho}{\partial S}\right]_{T} \delta S(E)
$$

in which

$$
\delta S(E) \simeq \frac{\chi \Delta \epsilon_{o} E^{2}}{24 \pi}
$$

where $\chi$ is the relevant susceptibility for the orientational order and $\Delta \epsilon_{o}$ the dielectric anisotropy of the medium with perfect orientational order $(S=1)$. The calculated phase diagram shows that the $\mathrm{A}_{d}$ phase is indeed bounded as a function of field (Fig.13). The increase in density favours the parallel pairs which have 
a lower McMillan parameter and as such the $A_{d}$ phase gets destablised as a function of field. Indeed it is well known that the $\mathrm{A}_{d}$ phase is also bounded as a function of pressure which compresses the medium [28].

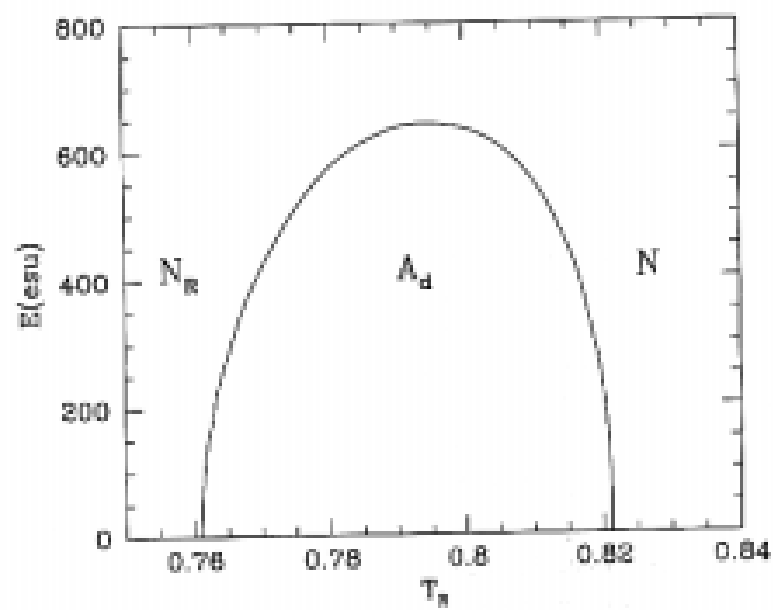

Figure 13. Calculated electric field phase diagram showing that of the $\mathrm{A}_{d}$ phase gets bounded $\left(\mathrm{R}_{1}=6, \mathrm{R}_{2}=0.8 \alpha_{A}=\right.$ 0.96 ) [27].

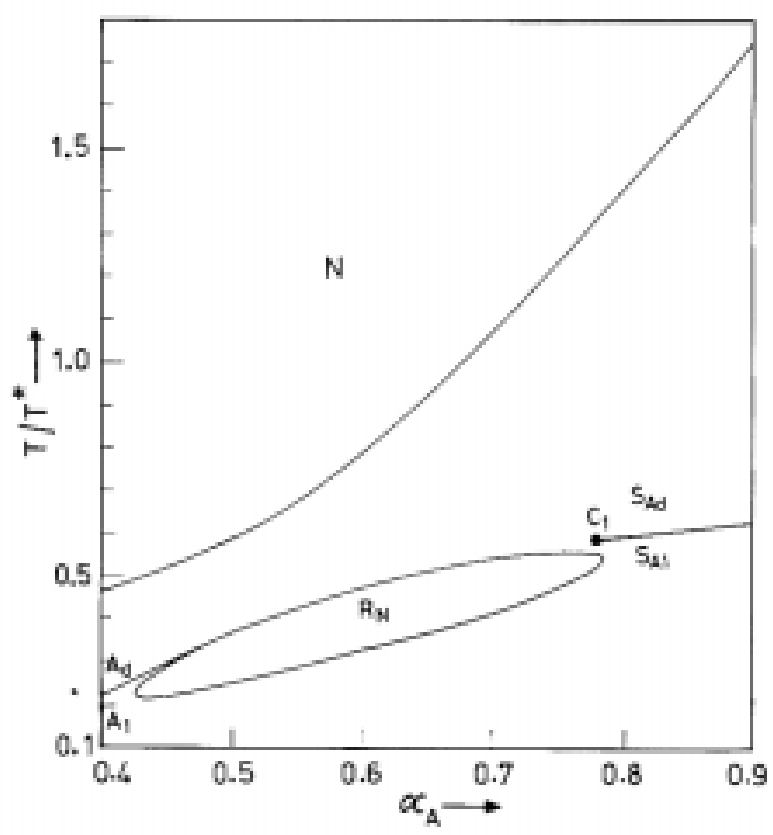

Figure 14. Calculated phases diagram showing a wide reentrant nematic $\left(\mathrm{R}_{N}\right)$ lake [29]. $\mathrm{C}_{1}$ indicates the critical point for the $\mathrm{A}_{1}-\mathrm{A}_{d}$ transition.

The model has been extended by taking into account the dependence of the interaction parameter $R_{1}$ on chain length i.e, the McMillan parameter $\alpha$ to get various other results. One of these is a first order $A_{1}$ to $\mathrm{A}_{d}$ transition which ends in a critical point as a function of $\alpha$, beyond which there is a continuous evolution of $\mathrm{A}_{1}$ to $\mathrm{A}_{d}$ in view of the fact that both states have the same symmetry. For an appropriate set of parameters, a reentrant nematic lake surrounded by the smectic A on all sides is also obtained (Fig. 14)[29]. Such phase diagrams have been studied both experimentally [30] and in the context of the Landau theory [1].

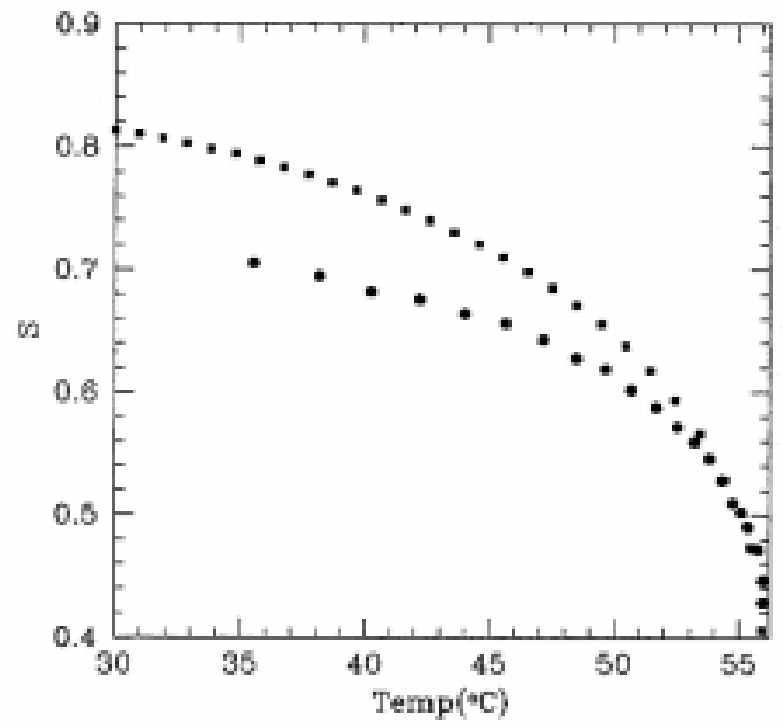

Figure 15. Comparison between the orientational order parameter measured from magnetic susceptibility (filled circles) and the effective order parameter $\mathrm{S}_{e}$ calculated from the dielectric constant $\epsilon_{11}$ (filled squares) in CP7B. The dielectric data has been normalised at $\mathrm{T}_{N I}-\mathrm{T}=2{ }^{\circ} \mathrm{C}$ with respect to the magnetic data. [31]

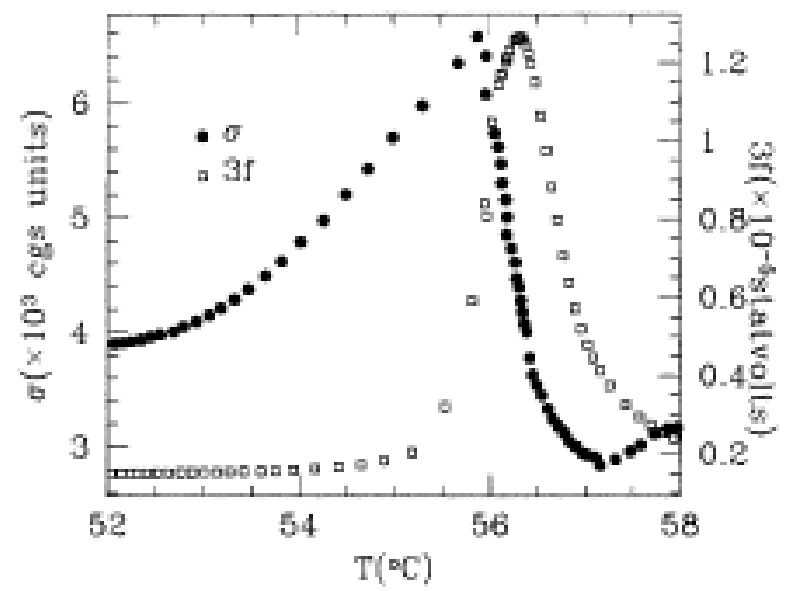

Figure 16. Temperature variations of the effective conductivity and third harmonic signal of CP7B of a $12 \mu \mathrm{m}$ thick sample under an applied field of 472 esu [31].

Very recent high field experiments conducted on pcyanophenyl-p-n-heptyl benzoate, (CP7B) which has an ester group whose dipole adds to that of the cyano group have yielded many new results which are is broad 
accord with the predictions of the molecular model discussed earlier [31]. The effective orientational order parameter calculated using low field dielectric constant measurements are much higher than those obtained from magnetic susceptibility measurements (Fig. 15). The difference increases at lower temperatures clearly implying that the short range order becomes more polar in nature, which would result from the increase in the relative concentration of parallel pairs at lower temperatures. When an electric field of $\sim 470 \mathrm{esu}$ which is close to the critical field for the nematic to field induced paranematic transition is applied to the cell, the effective conductivity of the sample shows an unusually large peak (Fig.16). Such a large peak is not seen near the critical temperatures of binary liquid mixtures, for example [32]. The peak has a characteristic asymmetry, and significantly, it occurs at a temperature lower than the critical temperature indicated by the peak in the third harmonic signal which is proportional to the susceptibility of the sample. The observations can be accounted for by assuming that relaxation of the polar short range ordered groups contribute to the dielectric loss and hence to the effective conductivity. The corresponding polarization will have a collective response as the critical point is approached. The Landau-Khalatnikov model [32] predicts that the relaxation rate for the collective response

$$
\frac{1}{\tau} \propto\left(\frac{T_{C}-T}{T_{C}}\right) .
$$

The relevant contribution to the conductivity is given by

$$
\sigma_{11}(w)=\frac{\epsilon_{o}\left(\delta \epsilon_{11}\right)}{1+w^{2} \tau^{2}} \tau w^{2}
$$

where $\epsilon_{o}$ is the vacuum dielectric constant and $\delta \epsilon_{11}$ the difference between the low frequency and high frequency dielectric constants. The peak of conductivity occurs at $w \tau=1$ where $w=2 \pi \nu$ is the angular frequency of the applied field. The experimental data could be fitted to these equations (Fig.17), with $\delta \epsilon_{11} \sim$ 3 which is much smaller than $\epsilon_{11}-\mu_{e}^{2} \simeq 20$, where $\mu_{e}$ is the extraordinary refractive index. This shows that only the fraction with polar short range order contributes to the relaxation process, as expected. Indeed a weak but nonzero second-harmonic peak is also seen at a high field which clearly indicates that there are po- larized domains which are unable to reorient with the field (Fig.18). These experimental results provide the first evidence for a polar short range order in highly polar compounds and lend support to the molecular theory outlined earlier.

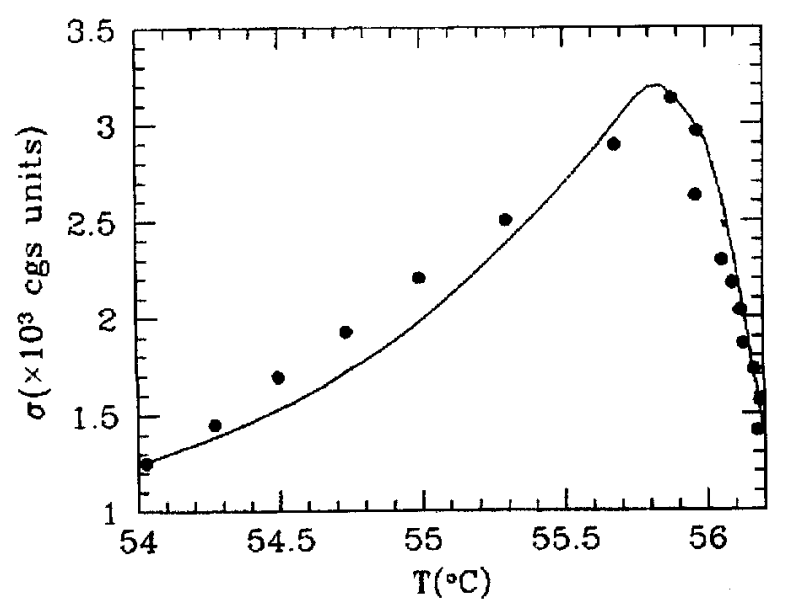

Figure 17. The fluctuation dependent part of the conductivity compared with the theoretical variation assuming a Landau-Khalatnikov slowing down of polarized domains [31].

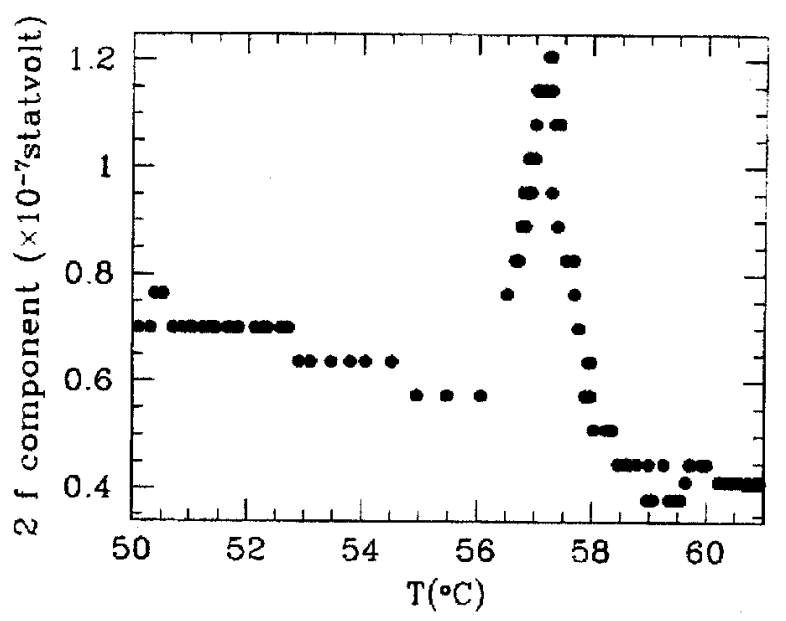

Figure 18. Peak in the second harmonic signal in a $10 \mu \mathrm{m}$ thick sample of CP7B under a field of $\sim 600 \mathrm{esu}$.

Another interesting observation on this compound is that at $600 \mathrm{esu}$ the intensity of light scattering shows a sudden increase as the temperature is lowered to $\sim$ $32.5^{\circ} \mathrm{C}$. Microscopic observations did not indicate either a biaxial nematic or a smectic A phase at lower temperatures. Hence it would appear that the medium exhibits a nematic to nematic transition in which the relative concentration of antiparallel pairs suddenly jumps across a weak first order transition. Indeed such a possibility had already been discussed as an extension of 
the $A_{1}-A_{d}$ transition line to the reentrant nematic range in the framework of the Landau theory [1]. Further an experimental evidence for such a transition has been found recently in one system [33]. Indeed in some parameter range of our molecular model, we had predicted such an N-N transition even in the absence of an associated $\mathrm{A}_{1}-\mathrm{A}_{d}$ transition [34]. The compound that we have studied is a pure nematogen and we have very recently found that even in the absence of an electric field, a thin $(\sim 3 \mu \mathrm{m})$ sample clearly exhibits a jump in the optical path difference which implies an N-N transition (Fig.19 ) [35]. Detailed measurements have shown that in such thin cells, the surface interaction leads to an increase of order parameter by $\sim 15 \%$, which would in turn enhance the $\mathrm{N}-\mathrm{N}$ transition temperature which probably has a subambient value in the bulk. The high electric field also has similar effects on the order parameter and the N-N transition point.

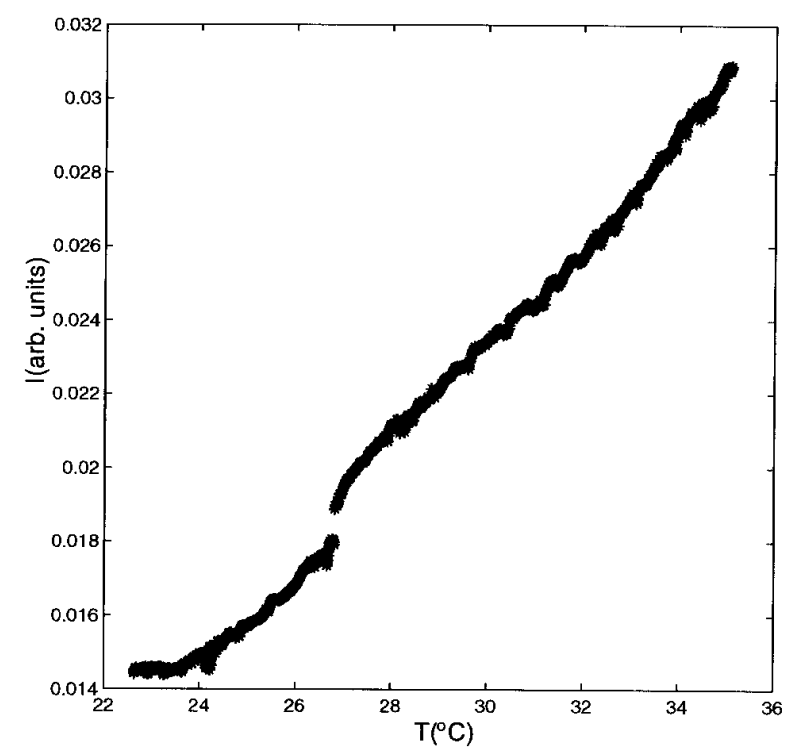

Figure 19. The transmitted intensity of a $3 \mu \mathrm{m}$ thick planar aligned sample of CP7B between two crossed polarizers set at $45^{\circ}$ to the director. Note the jump at $27^{\circ} \mathrm{C}$, indicating a weak first order $\mathrm{N}-\mathrm{N}$ transition.

\section{Conclusion}

In conclusion very high longitudinal dipoles make important contributions to intermolecular interactions and give rise to new types of liquid crystals like smectics with partial bilayer structure $\left(\mathrm{A}_{d}\right)$ and antiphase $\tilde{A}$, etc. Further, unusual phase sequences like those involving reentrant phases, smectic A- and even nematic- polymorphism are generated in such materials. We have presented arguments and experimental evidence to demonstrate that the molecules associate to form antiparallel pairs at moderate densities while at high densities, they prefer a parallel alignment, the latter structure giving rise to a polar short range order. An obviously interesting question is if some materials can exhibit polar long range order to give rise to a ferroelectric nematic as proposed by Born. Indeed there have been a large number of theoretical studies and computer simulations on polar fluids indicating that this is a clear possibility [36]. Even longitudinal ferroelectric smectics have been predicted to occur [37], though not realised as yet. Transverse ferroelectrics in both chiral [38] and nonchiral [39] liquid crystals are of course well established and are topics of vigorous research activity. Hopefully longitudinal ferroelectrics will also be discovered in the near future.

\section{References}

[1] P.G. de Gennes and J. Prost, The Physics of Liquid Crystals, second edition Clarendon Press, Oxford (1993).

[2] S. Chandrasekhar, Liquid Crystals, second edition, Cambridge University Press, Cambridge (1992).

[3] M. Born, Sitz d. Phys. Math. 25, 614 (1916).

[4] W. Maier and A. Saupe, Z. Naturforschg., 13a, 564 (1958).

[5] M. Schadt and W. Helfrich, Appl. Phys. Lett. 18, 127 (1971).

[6] M. Schadt, J. Chem. Phys. 56, 1494 (1972).

[7] N.V. Madhusudana, B.S. Srikanta and M. Subramanya Raj Urs, Mol. Cryst. Liquid Cryst. 108, 19 (1984)

[8] N.V. Madhusudana and S. Chandrasekhar, in Proceedings of the International liquid crystals conference, Bangalore, December 1973, Pramana Supplement 1, 57.

[9] N.V. Madhusudana, K.L. Savithramma and S. Chandrasekhar, Pramana, 8, 22 (1977).

[10] A.J. Leadbetter, R.M. Richardson and C.N. Colling, J.de Physique, 36, C1-37 (1975).

[11] P.E. Cladis, Phys. Rev. Lett. 35, 48 (1975).

[12] P.E. Cladis, Mol. Cryst. Liquid. Cryst. 165, 85 (1988).

[13] N.V. Madhusudana, B.K. Sadashiva and K.P.L. Moodithaya, Curr. Science 48, 613 (1979).

[14] F. Hardouin, G. Sigaud, M.F. Achard and H. Gasparoux, Solid State Commun. 30, 265 (1979).

[15] F. Hardouin and A.M. Levelut, J. de Physique, 41, 41 (1981). 
[16] H.T. Nguyen, F. Hardouin and C. Destrade, J. de Physique 43, 1127 (1982).

[17] G. Sigaud, F. Hardouin, M.F. Achard and H. Gasparoux, J. de Physique 40, C3-356 (1979).

[18] N.V. Madhusudana, B.S. Srikanta and Subramanya Raj Urs, Mol. Cryst. Liquid. Cryst. Lett. 82, 317 (1982).

[19] A.M. Levelut, R.J. Tarento, F. Hardouin, M.F. Achard and G. Sigaud, Phys. Rev. A 24, 2180 (1981).

[20] J. Prost, in Liquid crystals of one-and two-dimensional order, (eds) W. Helfrich and G. Heppke (Springer Verlag, Berlin, 1980) p. 125.

[21] T.R. Bose, C.D. Mukerjee, M.K Roy and M. Saha, Mol. Cryst. Liquid. Cryst. 126, 197 (1985)

[22] L. Longa and W.H. de Jeu, Phys. Rev. A 26, 1632 (1982).

[23] J.O. Indeku and A.N. Berker, J. de Physique, 49, 353 (1988).

[24] N.V. Madhusudana and Jyotsna Rajan, Liquid Crystals 7, 31 (1990).

[25] Geetha Basappa and N.V. Madhusudana, Mol. Cryst. Liquid. Cryst. 288, 161 (1996).

[26] I. Lelidis, M. Nobili and G. Durand, Phys. Rev. E 48, 3822 (1993)

[27] Geetha Basappa, A.S. Govind and N.V. Madhusudana, J. de Physique, II7, (1997).
[28] P.E. Cladis, Mol. Cryst. Liquid. Cryst 67, 177 (1981).

[29] A.S. Govind and N.V. Madhusudana, Liq. Cryst. 23, 327 (1997).

[30] F. Hardouin, M.F. Achard, N.H. Tinh and G. Sigaud, Mol. Cryst. Liquid. Cryst. Lett. 3, 7 (1986).

[31] Geetha Basappa and N.V. Madhusudana, Eur. Phys. J. B 1, 179 (1998).

[32] M.A. Anisimov, Critical Phenomena in Liquids and Liquid Crystals (Gordon and Breach Science Publishers), 1991.

[33] G. Nounesis, S. Kumar, S. Pfieffer, R. Shashidhar and C.W. Garland, Phys. Rev. Lett. 73565 (1994).

[34] A.S. Govind and N.V. Madhusudana, Liq. Cryst. 14 1539 (1993).

[35] Sobha Warrier, D. Vijayaraghavan and N.V. Madhusudana, Europhys. Letters (in press).

[36] B. Groh and S. Dietrich, Phys. Rev. Lett. 722422 (1994)

[37] D.R. Perchak and R.G. Petschek, Phys. Rev. A 43, 6756 (1991).

[38] R.B. Meyer, L. Liebert, L. Stzelecki and D. Keller, J. de Physique. Lett. 36, L69 (1975)

[39] T. Niori, T. Selkine, J. Watanabe, T. Furokawa and H. Takezoe, Mol. Cryst. Liquid Cryst. 301, 337 (1997). 